\title{
BMJ Open Statin prescribing for people with severe mental illnesses: a staggered cohort study of 'real-world' impacts
}

\author{
R Blackburn, ${ }^{1}$ D Osborn, ${ }^{2}$ K Walters, ${ }^{3}$ M Falcaro, ${ }^{3}$ I Nazareth, ${ }^{3}$ I Petersen ${ }^{3}$
}

To cite: Blackburn $\mathrm{R}$, Osborn D, Walters K, et al. Statin prescribing for people with severe mental illnesses: a staggered cohort study of 'real-world' impacts. BMJ Open 2017;7:e013154. doi:10.1136/bmjopen-2016013154

- Prepublication history and additional material is available. To view please visit the journal (http://dx.doi.org/ 10.1136/bmjopen-2016013154).

Received 23 June 2016 Revised 1 December 2016 Accepted 14 February 2017

CrossMark

${ }^{1}$ Institute for Health Informatics, UCL, London, UK

${ }^{2}$ Division of Psychiatry, UCL, London, UK

${ }^{3}$ Primary Care and Population Health, UCL, London, UK

Correspondence to Dr R Blackburn; r.blackburn@ucl.ac.uk

\section{ABSTRACT}

Objectives: To estimate the 'real-world effectiveness of statins for primary prevention of cardiovascular disease (CVD) and for lipid modification in people with severe mental illnesses (SMI), including schizophrenia and bipolar disorder.

Design: Series of staggered cohorts. We estimated the effect of statin prescribing on CVD outcomes using a multivariable Poisson regression model or linear regression for cholesterol outcomes.

Setting: 587 general practice (GP) surgeries across the UK reporting data to The Health Improvement Network.

Participants: All permanently registered GP patients aged 40-84 years between 2002 and 2012 who had a diagnosis of SMI. Exclusion criteria were pre-existing CVD, statin-contraindicating conditions or a statin prescription within the 24 months prior to the study start. Exposure: One or more statin prescriptions during a 24-month 'baseline' period (vs no statin prescription during the same period).

Main outcome measures: The primary outcome was combined first myocardial infarction and stroke. All-cause mortality and total cholesterol concentration were secondary outcomes.

Results: We identified 2944 statin users and 42886 statin non-users across the staggered cohorts. Statin prescribing was not associated with significant reduction in CVD events (incident rate ratio 0.89; $95 \%$ $\mathrm{Cl} 0.68$ to 1.15$)$ or all-cause mortality $(0.89 ; 95 \% \mathrm{Cl}$ 0.78 to 1.02 ). Statin prescribing was, however, associated with statistically significant reductions in total cholesterol of $1.2 \mathrm{mmol} / \mathrm{L}(95 \% \mathrm{Cl} 1.1$ to 1.3$)$ for up to 2 years after adjusting for differences in baseline characteristics. On average, total cholesterol decreased from 6.3 to 4.6 in statin users and 5.4 to $5.3 \mathrm{mmol} / \mathrm{L}$ in non-users.

Conclusions: We found that statin prescribing to people with SMI in UK primary care was effective for lipid modification but not CVD events. The latter finding may reflect insufficient power to detect a smaller effect size than that observed in randomised controlled trials of statins in people without SMI.

\section{INTRODUCTION}

People with severe mental illness (SMI) including schizophrenia and bipolar disorder
Strengths and limitations of this study

- We used a large and representative data source that records real-world clinical data on cardiovascular outcomes and lipid modification in people with severe mental illness.

- Such data are both costly and difficult to obtain from other data sources and recruitment of people with severe mental illness into clinical trials may be challenging and result in substantial selection bias.

- We applied specialist methods to reduce the impact of confounding by indication (staggered cohort and multivariable regression) and handle missing covariate data (multiple imputation) within our study.

- However, these methods can only reduce bias arising from differences in measured confounders. Residual confounding may remain due to unmeasured confounders such as diet or severity of mental illness.

- We were not able to examine the association between statin prescribing and lipid modification among people who had missing data on total cholesterol at baseline.

are at a twofold to threefold higher risk of cardiovascular disease (CVD) than comparable individuals without SMI. ${ }^{12}$ CVD drives a substantial portion of the 13-30 years deficit in life expectancy relative to the general population $^{3}$ and National Institute for Health and Care Excellence (NICE) guidelines identify people with SMI as a population for which statin prescribing should be considered. $^{4-6}$ Statins are cost-effective for preventing CVD events within randomised controlled trial (RCT) populations without mental illness. ${ }^{7}$ However, we do not know whether statins are similarly effective in people with SMI, since this population has differences in CVD risk profile (such as very high rates of smoking, obesity and diabetes). Furthermore, people with SMI may adhere to medication differently and have additional exposure to antipsychotic 
medications, which are associated with increased risk of dyslipidaemia.

We do not know if results from existing RCTs can be extrapolated to real-world impacts of statin prescribing to people with SMI. Over two-thirds of the participants in the most recent meta-analysis of statins for primary prevention of $\mathrm{CVD}^{7}$ were derived from studies that explicitly excluded participants with psychological conditions $(50 \% ; n=28390)^{8-10}$ or individuals who were perceived as less likely to be compliant with treatment $(20 \% ; \mathrm{n}=10797) .^{11-13}$

Few studies have assessed statin medication adherence in people with SMI and we are unaware of any that focus exclusively on primary CVD prevention. However, a small number of important studies have investigated cardiovascular medication adherence for combined primary and secondary prevention and report comparable or better adherence for people with schizophrenia relative to controls. ${ }^{14}{ }^{15}$ Despite such findings, mental illness is sometimes perceived as a barrier to good medication adherence ${ }^{16-18}$ and might therefore deter general practitioners from prescribing statins to people with SMI. ${ }^{19}$ Indeed, significant inequalities in statin prescribing have been reported for people with SMI. ${ }^{20}$ In addition, in vitro studies indicate that some antipsychotic agents interact with transcription factors for enzymes involved in cholesterol and fatty acid synthesis (including 3-hydroxy-3-methylglutaryl-coenzyme A (HMG-CoA) reductase, the primary binding site for statins) and could therefore counteract the cholesterol-lowering action of statins. ${ }^{21} 22$ The degree of interaction appears to vary by type of antipsychotic and is more strongly correlated with agents such as clozapine, which are associated with weight gain. ${ }^{23} 24$

We aimed to estimate the real-world impact of statin prescribing on people with SMI by comparing people with SMI who did (statin users), or did not (statin non-users), receive a statin prescription. We estimated the effect of statin prescribing on:

1. Combined first myocardial infarction (MI) and stroke (primary outcome);

2. All-cause mortality;

3. Change in total cholesterol concentration at 1 and 2 years after initiating a statin.

\section{METHODS}

\section{Study design}

We used data from The Health Improvement Network (THIN), which captures anonymised data from electronic health records from over 12 million patients registered at 587 general practice (GP) surgeries $(\sim 5.7 \%$ of the UK population). ${ }^{25}$ See end of article for details of ethical approval. This data source has been used extensively to investigate CVD risk in people with SMI. ${ }^{1}{ }^{26} 27$ Individuals with SMI routinely access primary care ${ }^{28}$ and the validity of SMI diagnoses in computer records has been established. ${ }^{29}$ Furthermore, the incidence of SMI has been found to be comparable to other epidemiological studies. ${ }^{30}$ THIN data are recorded as hierarchical medical codes (Read codes), free-text comments, drug codes for prescribed medications, referrals and additional health information such as laboratory test results. ${ }^{31}{ }^{32}$ The Townsend score for the quintile of deprivation for a patient's address reflects deprivation (employment, household occupancy, car and home ownership as recorded in the 2001 census) at enumeration district level, which covers areas of $\sim 150$ households. ${ }^{33}$

Classification criteria (including case definitions for SMI, CVD and statin prescribing) for this study are outlined in online supplementary file S1 and code lists are available on request from the authors. We included all people with a diagnosis of schizophrenia or bipolar disorder who were aged 40-84 years and consulted their GP between 1 January 2002 and 31 December 2013. We excluded patients who were not permanently registered, and data for time periods before the practice acceptable mortality rate, acceptable computer usage dates ${ }^{34} 35$ and for the 12 months after patient registration. ${ }^{36}$ We excluded individuals who-before the cohort start-had any of: CVD diagnosis, statin prescription in the prior 24 months, condition likely to impact on initiating a statin (terminal illness, dementia or raised alanine transaminase and aspartate transaminase; defined as $>3 \mathrm{x}$ upper limit of normal range; 124 and $116 \mathrm{IU} / \mathrm{L}$, respectively).

As described more fully in our study protocol (see online supplementary file S2), we used a staggered cohort study design to reduce the impact of confounding by indication. ${ }^{37}$ This type of study design has successfully been applied to other studies comparing an active (including statins for primary CVD) and passive treatment groups. ${ }^{38-40}$

We used THIN data to create five 'staggered' cohort studies with 2-year follow-up periods between 1 January 2002 and 1 January 2010 (figure 1). Further detail regarding the selection of the time window is outlined in online supplementary file S3. We assessed the effectiveness of statin prescribing using pooled data from these cohorts. Each cohort study compared statin user and nonstatin user comparator groups, which were defined by whether or not an individual was prescribed a statin (one or more prescriptions) during the first 24 months of the study start. Statin users and non-users were redefined at the start of each separate staggered cohort.

\begin{tabular}{|c|c|c|c|c|c|}
\hline Jan 02 & Jan 04 & Jan 06 & Jan 08 & Jan 10 & Dec 13 \\
\hline statin & \multirow{2}{*}{\multicolumn{5}{|c|}{ follow up }} \\
\hline \multirow{9}{*}{ no statin } & & & & & \\
\hline & statin & follow un & & & \\
\hline & no statin & $1030 \mathrm{vo}$ aps & & & \\
\hline & & statin & follow up & & \\
\hline & & no statin & & & \\
\hline & & & statin & 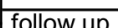 & \\
\hline & & & no statin & & \\
\hline & & & & statin & follow up \\
\hline & & & & no statin & \\
\hline
\end{tabular}

Figure 1 Diagrammatic representation of the staggered cohort study design. 
Statin users began follow-up on the day of their first statin prescription (the index date); for non-statin users, the index date was a randomly selected day within the 2-year exposure period. Statin users and non-users who had a CVD event recorded within 3 months of the index date were excluded because this pattern may reflect delayed recording of the CVD event, where the statin was initiated for secondary prevention of CVD. The end of follow-up was first CVD event, death or loss to follow-up (out of practice transfer or end of the study period).

The primary outcome was first MI or stroke (combined MI, haemorrhagic and ischaemic or unspecified stroke). Secondary outcomes were all-cause mortality, MI, stroke, change in total cholesterol level/concentration (for those with available records) 1 and 2 years after the index date. We selected these outcomes because other studies have identified high positive predictive values (85-95\% for CVD events and $99 \%$ for allcause mortality) for MI, ${ }^{41}{ }^{42}$ stroke $^{43}{ }^{44}$ and all-cause mortality ${ }^{45}$ in THIN and closely related databases. We also considered investigating coronary heart disease (CHD) as an outcome, but feasibility work suggested that some CHD Read codes such as 'unspecified CHD' may reflect retrospective recording of pre-existing CHD.

We extracted data on covariates recorded during the 12 months before the index date (see online supplementary files S1 and S3). Covariates included age, sex, type of SMI, blood pressure, weight, height, cholesterol concentration, diabetes, smoking status, Townsend score, antihypertensive use, non-statin lipid modification (eg, fibrates), heavy alcohol drinking, familial hypercholesterolaemia, hypothyroidism, chronic kidney disease, chronic obstructive pulmonary disease, asthma, atrial fibrillation, predominant antipsychotic, mood stabilising drug, antidepressant drug, annual consultation rate and cancer diagnosis.

Unobserved covariate data (total cholesterol, height, weight, systolic blood pressure and smoking status) were estimated for the full study population using multiple imputation with chained equations to generate 10 imputed data sets. ${ }^{46}$ Data were separately imputed by gender in each of the five cohorts and used covariate data measured within 3 years of baseline. CVD events and Nelson-Aalen estimates of the cumulative hazard function for the time to event were included in the imputation model. ${ }^{47} \mathrm{~A}$ detailed description of the model and subsequent data checks is outlined in online supplementary file S3.

\section{Analysis}

Data for each of the five cohort studies were pooled to obtain average estimates for the whole study period. Incident rate ratios (IRRs) for the association between statin prescribing and combined MI and stroke were estimated using a Poisson regression model with receipt of a statin prescription as the exposure and first MI or stroke event as the outcome: the log of follow-up time was used as an offset. Since the same individual could be included in more than one cohort, the 'robust sandwich estimator' was used to calculate conservative estimates of variance. ${ }^{48}$ The model was adjusted for covariates outlined previously. We also assessed the impact of log-transforming continuous variables and including interaction terms for age and sex.

All analyses were undertaken using an intentionto-treat approach with exposure defined by statin prescribing at the index date. The primary analysis was repeated for: all-cause mortality (death from any cause), first stroke (haemorrhagic or ischaemic stroke) and first MI to identify whether the effectiveness of statins was substantially altered for each of these outcomes. Changes in total cholesterol concentration measured during the 1 and 2 years after the index date (ie, 1-365 or 366-730 days after the index date, respectively) were evaluated for statin users and non-users in individuals with complete data at baseline. For this analysis, linear regression models with total cholesterol at 1 or 2 years after the index date were developed using the same array of covariates as for the main analysis. Change in cholesterol as an outcome for individuals who did not have complete data was not assessed. Data management and analysis were undertaken in Stata V.14.

\section{RESULTS}

Overall, 45830 participants (defined as total statin users and non-users under follow-up) were included in the pooled data set. These participants reflect data for 16854 individuals with schizophrenia or bipolar disorder who, on average, were included in 2.7 of the five possible cohorts. A total of 25 statin users and 27 nonstatin users were excluded because they had a CVD event recorded within 3 months of the index date. A flow chart outlining numbers of statin users and non-users in and the associated event rate for each of the five cohort studies is outlined in figure 2. Table 1 describes the baseline characteristics of statin users and non-users for the full study population (using imputed data) compared with the subset with fully observed covariate data at baseline. Of note, total cholesterol concentration at baseline differed in statin users and non-users. The distribution of estimated CVD risk scores was different for statin users and non-users, with baseline risk being higher (on average) for statin users. Statin users also had greater levels of deprivation, consulted more frequently and had higher frequencies of prescribingincluding antihypertensives and antidepressants-and to have familial hypercholesterolaemia (for which statin therapy is indicated; table 1).

Modelled associations between covariates and statin prescribing or CVD events are outlined in the supplementary material for complete cases (see online supplementary file 4 , table S1) and the imputed data set (see online supplementary file 4, table S2).

The outputs of each of the regression models outlined in the results are included as a supplementary file (see online supplementary file 4). 


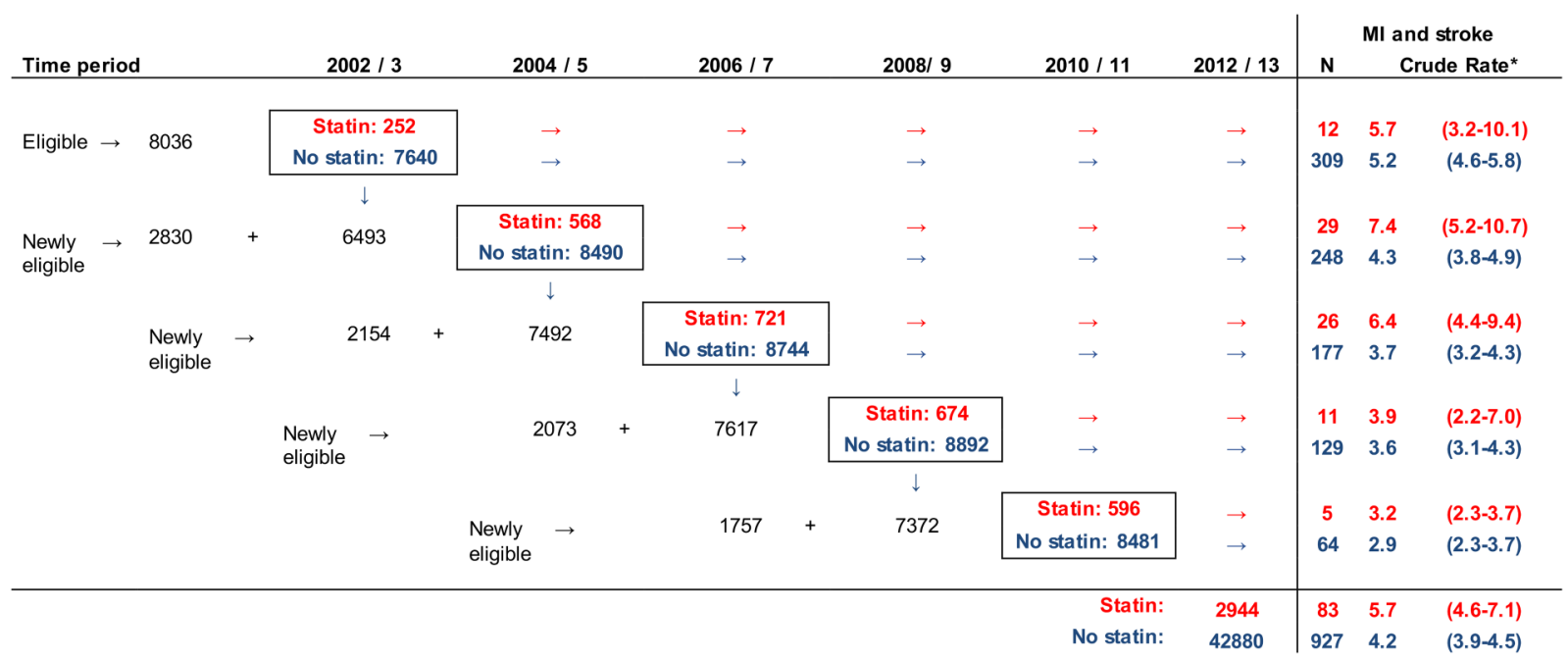

*Crude event rates (and associated $95 \% \mathrm{Cl}$ ) per 1000 person-years

This figure describes the flow of individuals between adjacent cohorts (e.g. 6493 individuals who were statin non-users in $2002 / 3$ were included in the $2004 / 5$ cohort in addition to 2830 newly eligible individuals). Of the 2944 statin prescriptions issued at the index date, 2938 ( $83 \%$ ) were for simvastatin, $395(13 \%)$ for atorvastatin and the remaining $111(4 \%)$ for pravastatin $(n=46)$, rosuvastatin $(n=45)$, fluvastatin $(n=16)$ or cerivastatin $(n=4)$.

Figure 2 Flow chart of statin users and non-users contributing data to each of the five cohorts in the full population. MI, myocardial infarction.

The crude rate of first MI and stroke per 1000 personyears of follow-up was 5.74 (95\% CI 4.62 to 7.11 ) in statin users and 4.17 (95\% CI 3.91 to 4.45$)$ in statin non-users in the full population (further outlined in figure 2). The crude IRR for the association between statin prescribing and MI and stroke events was 1.39 (95\% CI 1.11 to 1.74) for the full study population: which was reduced to 0.89 (95\% CI 0.68 to 1.15 ) after adjustment for all additional covariates (see online supplementary file 4, table S3). Inclusion of quadratic or log-transformed versions of continuous variables (age, total cholesterol, systolic blood pressure, body mass index) in the model did not have a marked impact on the results (data not shown).

The crude IRR for the association between statin prescribing and all-cause mortality was 1.02 (95\% CI 0.90 to 1.14 ) and was reduced to 0.89 (95\% CI 0.78 to 1.02 ) after adjusting for all covariates as above (see online supplementary file 4 , table S3).

The crude IRR for the association between statin prescribing and first MI was 1.51 (95\% CI 1.04 to 2.18) and was reduced to 0.75 (95\% CI 0.48 to 1.15$)$ after adjusting for all covariates (see online supplementary file 4 , table S3).

The crude IRR for the association between statin prescribing and first stroke was 1.31 (95\% CI 0.99 to 1.74 ) and was reduced to 0.96 (95\% CI 0.68 to 1.15 ) after adjusting for all covariates (see online supplementary file 4 , table S3).

A total of $82 \%$ and $73 \%$ of the 1714 statin users had measurements of total cholesterol recorded within the 1 and 2 years after the index date, respectively. By comparison, $55 \%$ and $57 \%$ of statin non-users had a measurement of cholesterol recorded within the 1 and 2 years after the index date, respectively.

In statin users, total cholesterol decreased on average by $1.7 \mathrm{mmol} / \mathrm{L}(27 \%)$ from baseline concentrations of $6.3-4.6 \mathrm{mmol} / \mathrm{L}$ at both 1 and 2 years after baseline (table 2). In contrast, among statin non-users, mean total cholesterol in each individual was slightly decreased by $0.1 \mathrm{mmol} / \mathrm{L}(2 \%)$ from baseline concentrations of $5.4-5.3 \mathrm{mmol} / \mathrm{L}$ at both 1 and 2 years after baseline (table 2).

The adjusted linear regression model showed a mean reduction in total cholesterol among statin users (relative to non-users) of $1.3 \mathrm{mmol} / \mathrm{L}$ (95\% CI 1.2 to 1.4 ; see online supplementary file 4 , table S4) and $1.2 \mathrm{mmol} / \mathrm{L}$ (95\% CI 1.1 to 1.3 ; see online supplementary file 4 , table S5) at 1 and 2 years after the index date, respectively. Although statin use was the most important predictor of change in cholesterol concentration at both time points, baseline cholesterol was also strongly associated with subsequent changes in total cholesterol (coefficients of -0.36 and -0.41 at 1 and 2 years, respectively; see online supplementary file 4 , tables $\mathrm{S} 4$ and S5, respectively).

\section{DISCUSSION}

\section{Principal findings}

Our results provide evidence that statin prescribing to people with SMI may carry a similar level of benefit to the general population with observed reductions for total cholesterol of $1.2 \mathrm{mmol} / \mathrm{L}$ for up to 2 years. While non-significant, the effect estimates for combined MI and stroke (IRR $0.89 ; 95 \%$ CI 0.68 to 1.15 ) and all-cause 
Table 1 Characteristics of the study population at baseline (for complete records and the full population after imputation of unobserved data)

\begin{tabular}{|c|c|c|c|c|c|c|c|c|}
\hline \multirow[b]{2}{*}{ Characteristic at baseline } & \multicolumn{4}{|l|}{ Complete cases } & \multicolumn{4}{|l|}{ Full population } \\
\hline & Statin non-user & IQR [or \%] & Statin user & IQR [or \%] & Statin non-user & IQR [or \%] & Statin user & IQR [or \%] \\
\hline Total participants under follow-up* & 5201 & & 1714 & & 42886 & & 2944 & \\
\hline Bipolar & 2612 & [50\%] & 835 & {$[49 \%]$} & 21883 & [51\%] & 1460 & [50\%] \\
\hline Median age (years) & 55 & $(48,64)$ & 58 & $(50,65)$ & 54 & $(47,64)$ & 59 & $(51,67)$ \\
\hline Male & 2438 & [47\%] & 866 & [51\%] & 19191 & [45\%] & 1400 & [48\%] \\
\hline Diabetes (yes) & 1156 & [22\%] & 943 & [55\%] & 3393 & {$[8 \%]$} & 1020 & [35\%] \\
\hline $\begin{array}{l}\text { Median systolic BP }(\mathrm{mm} \mathrm{Hg}) \dagger \\
\text { Per cent imputed }\end{array}$ & 131 & $(120,141)$ & 136 & $(125,147)$ & 130 & $\begin{array}{l}(120,141) \\
{[43 \%]}\end{array}$ & 137 & $\begin{array}{l}(126,148) \\
{[7 \%]}\end{array}$ \\
\hline $\begin{array}{l}\text { Median BMl† } \\
\text { Per cent imputed }\end{array}$ & 28 & $(25,33)$ & 30 & $(26,34)$ & 27 & $\begin{array}{l}(24,31) \\
{[62 \%]}\end{array}$ & 30 & $\begin{array}{l}(26,34) \\
{[30 \%]}\end{array}$ \\
\hline $\begin{array}{l}\text { Median cholesterol concentration }(\mathrm{mmol} / \mathrm{L}) \uparrow \\
\text { Per cent imputed }\end{array}$ & 5.3 & $(4.7,6.0)$ & 6.2 & $(5.5,7.1)$ & 5.4 & $\begin{array}{l}(4.8,6.2) \\
{[77 \%]}\end{array}$ & 6.4 & $\begin{array}{l}(5.6,7.2) \\
{[10 \%]}\end{array}$ \\
\hline & 3004 & [58\%] & 1120 & [65\%] & 23486 & {$[55 \%]$} & 1786 & {$[61 \%]$} \\
\hline \multicolumn{9}{|l|}{$\begin{array}{l}\text { Per cent imputed } \\
\text { Median Framingham CVD risk score (BMI) }\end{array}$} \\
\hline \multicolumn{9}{|l|}{ Women } \\
\hline $40-49$ years & 6 & $(4,10)$ & 10 & $(7,16)$ & 5 & $(4,7)$ & 10 & $(6,14)$ \\
\hline $50-59$ years & 11 & $(8,15)$ & 19 & $(12,26)$ & 10 & $(7,14)$ & 17 & $(11,24)$ \\
\hline \multirow{2}{*}{$\begin{array}{l}60-74 \text { years } \\
75-84 \text { years } \ddagger\end{array}$} & 20 & $(13,30)$ & 29 & $(19,42)$ & 17 & $(12,25)$ & 25 & $(17,38)$ \\
\hline & 34 & $(23,49)$ & 36 & $(27,56)$ & 26 & $(18,36)$ & 34 & $(24,48)$ \\
\hline \multicolumn{9}{|l|}{ Men } \\
\hline $40-49$ years & 14 & $(10,20)$ & 20 & $(15,28)$ & 12 & $(9,16)$ & 19 & $(14,26)$ \\
\hline \multirow{2}{*}{$\begin{array}{l}50-59 \text { years } \\
60-74 \text { years }\end{array}$} & 26 & $(18,35)$ & 33 & (2342) & 22 & $(16,30)$ & 32 & (2241) \\
\hline & 41 & $(29,55)$ & 47 & $(35,59)$ & 36 & $(26,47)$ & 45 & $(34,57)$ \\
\hline \multirow[t]{2}{*}{ 75-84 years $\ddagger$} & 58 & $(45,71)$ & 62 & $(48,80)$ & 49 & $(39,63)$ & 58 & $(48,79)$ \\
\hline & \multicolumn{4}{|l|}{ Complete cases } & \multicolumn{4}{|l|}{ Full population } \\
\hline Characteristic at baseline & Statin non-user & Per cent & Statin user & Per cent & Statin non-user & Per cent & Statin user & Per cent \\
\hline \multirow{2}{*}{\multicolumn{9}{|c|}{$\begin{array}{l}\text { Total participants under follow-up* } \\
\text { Number of GP consultations during baseline }\end{array}$}} \\
\hline & & & & & & & & \\
\hline$<4$ & 630 & $12 \%$ & 150 & $9 \%$ & 10906 & $25 \%$ & 322 & $11 \%$ \\
\hline $4-6$ & 1024 & $20 \%$ & 323 & $19 \%$ & 9310 & $22 \%$ & 568 & $19 \%$ \\
\hline $7-12$ & 1679 & $32 \%$ & 561 & $33 \%$ & 11506 & $27 \%$ & 942 & $32 \%$ \\
\hline$>12$ & 1868 & $36 \%$ & 680 & $40 \%$ & 11158 & $26 \%$ & 1112 & $38 \%$ \\
\hline Antipsychotic use & & & & & & & & \\
\hline None & 1926 & $37 \%$ & 649 & $38 \%$ & 20061 & $47 \%$ & 1178 & $40 \%$ \\
\hline First generation & 1091 & $21 \%$ & 384 & $22 \%$ & 8932 & $21 \%$ & 630 & $21 \%$ \\
\hline Second generation & 2184 & $42 \%$ & 681 & $40 \%$ & 13886 & $32 \%$ & 1136 & $39 \%$ \\
\hline Townsend score quintile & & & & & & & & \\
\hline 1 (least deprived) & 813 & $16 \%$ & 238 & $14 \%$ & 7280 & $17 \%$ & 448 & $15 \%$ \\
\hline 2 & 823 & $16 \%$ & 253 & $15 \%$ & 7419 & $17 \%$ & 462 & $16 \%$ \\
\hline 3 & 987 & $19 \%$ & 336 & $20 \%$ & 8653 & $20 \%$ & 595 & $20 \%$ \\
\hline
\end{tabular}




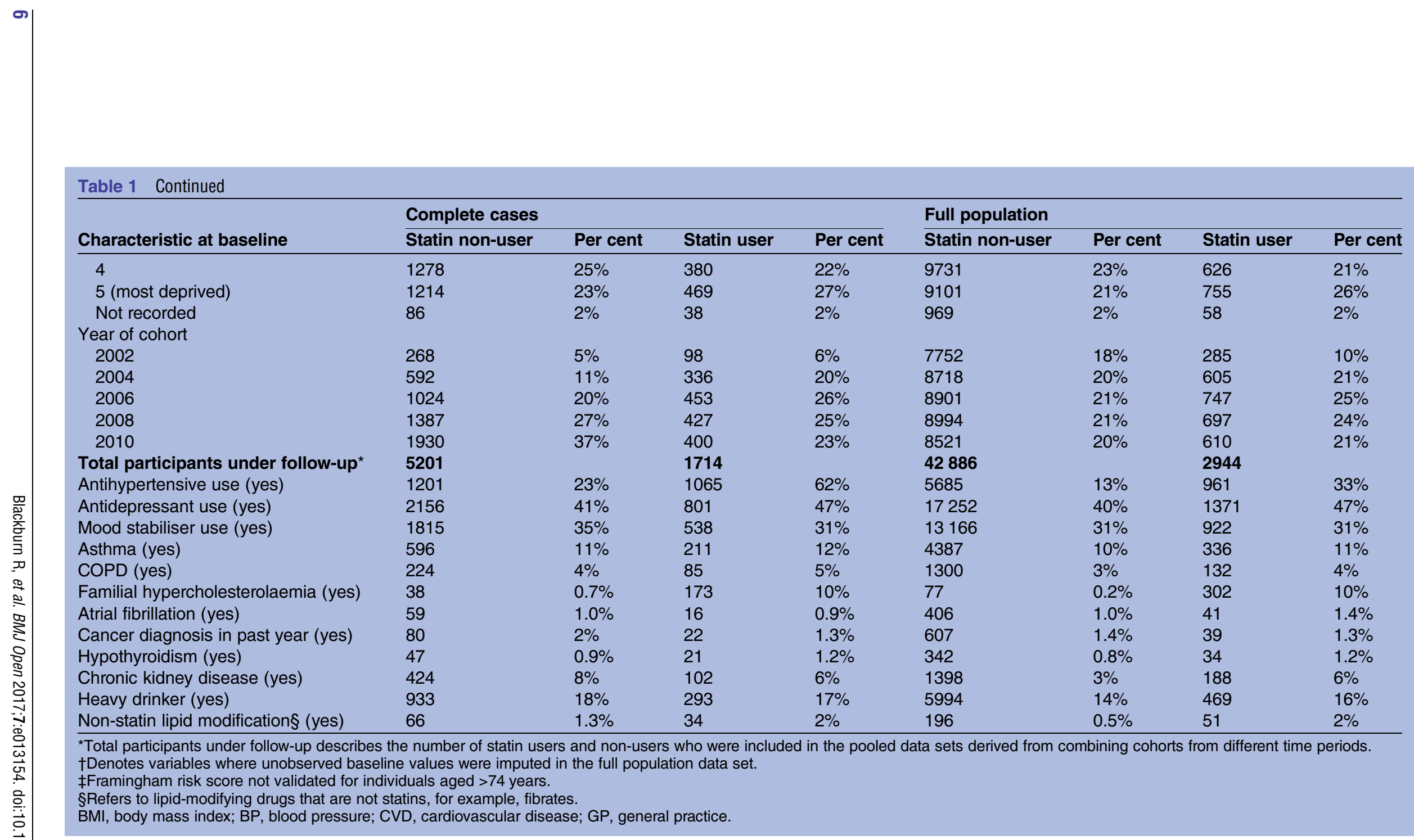


Table 2 Summary statistics for change in total cholesterol within 1 and 2 years of the index date

\begin{tabular}{lllll}
\hline Group & Description of metric & Baseline & $\mathbf{1}$ year & \multicolumn{2}{c}{$\mathbf{2}$ years } \\
\hline Statin non-users & Number under follow-up & 5201 & 2865 & 3002 \\
& Mean cholesterol concentration mmol/L (SD) & $5.4(1.0)$ & $5.3(1.0)$ & $5.3(1.0)$ \\
\multirow{3}{*}{ Statin users } & Change from baseline mmol/L (\%) & - & $-0.1(-2 \%)$ & $-0.1(-2 \%)$ \\
& Number under follow-up & 1714 & 1409 & 1253 \\
& Mean cholesterol concentration mmol/L (SD) & $6.3(1.2)$ & $4.6(1.0)$ & $4.6(1.1)$ \\
\hline
\end{tabular}

mortality ( 0.89 with $95 \%$ CI 0.78 to 1.02 ; figure 3 ) were broadly similar to published estimates from RCT meta-analysis ${ }^{7}$ and an externally validated observational study of people without SMI. ${ }^{49}$

\section{Comparison with other studies}

An IRR of 0.89 (with 95\% CI 0.68 to 1.15 ) is compatible with rates of MI and stroke being $11 \%$ lower among statin users than non-users within the study sample, but which - through chance alone-might not translate into a decreased event rate in the population. Thus, although the point estimates for our study are similar to observational studies of statins in the general population, ${ }^{49} 50$ we cannot conclude that statins are effective in preventing CVD events in people with SMI. Sample size estimation for this type of study design is not straightforward: however, Poisson counting error (see online supplementary file S5 for more detail) provides an indication that our study was likely to be adequately powered to detect a reduction in the rate of MI and stroke with a magnitude of effect similar to that observed in statin RCTs (22\% reduction) - but not observational studies (14\% reduction) -in people without $\mathrm{SMI}^{7}$ The estimated association between statin prescribing and allcause mortality (IRR of 0.89 ) was also similar to those derived from RCT meta-analysis ${ }^{7}$ and observational studies ${ }^{49}$ although scope for direct comparison is

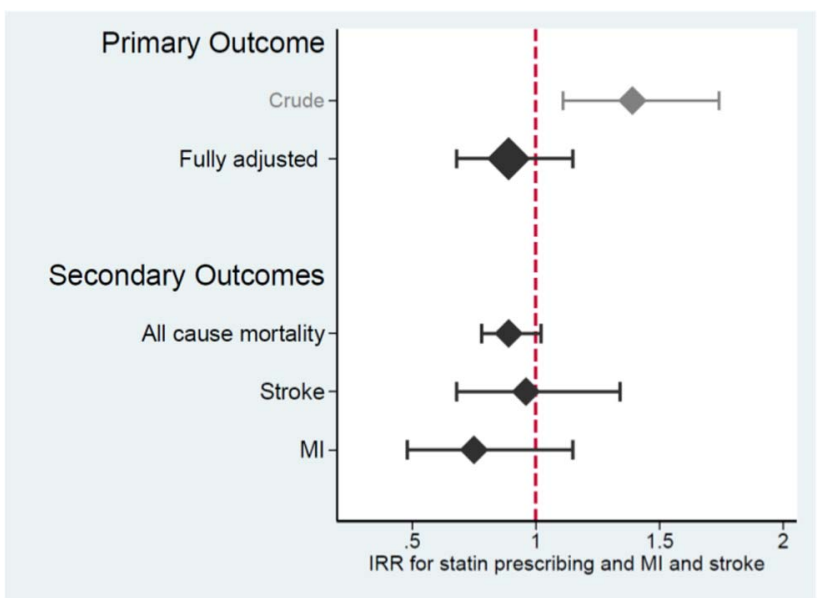

Figure 3 Key results for the association between statin prescribing and the rate of $\mathrm{Ml}$, stroke and all-cause mortality. $\mathrm{IRR}$, incident rate ratio; MI, myocardial infarction. limited by marked variations in mortality rate and duration of follow-up between our study and RCTs included in the meta-analysis. ${ }^{7}$

Statin use was associated with a mean decrease in total cholesterol concentration at 1 and 2 years after the index date of 1.3 and $1.2 \mathrm{mmol} / \mathrm{L}$, respectively (equivalent to a $20-21 \%$ decrease from the index date), after adjusting for differences in the baseline characteristics of statin users and non-users. RCTs with a minimum of 6 months of follow-up in people without SMI have identified a similar net decrease in total cholesterol of $1.05 \mathrm{mmol} / \mathrm{L}$ (95\% CI 0.76 to 1.35$)$, with variation between difference types and dosage of statin. ${ }^{7}$ Two other studies (that included a control group) have investigated statin prescribing in people with SMI. ${ }^{51}{ }^{52}$ A nonrandomised study compared changes in lipid levels during a 12-week period in 52 individuals taking $10 \mathrm{mg}$ rosuvastatin daily and 48 individuals not taking statins: total cholesterol concentration was decreased by an additional $2.9 \mathrm{mmol} / \mathrm{L}(35 \%)$ in statin users. ${ }^{51}$ A pilot study randomised 60 individuals to receive $40 \mathrm{mg}$ pravastatin daily or no statin for 12 weeks and reported smaller decreases in total cholesterol of $0.5 \mathrm{mmol} / \mathrm{L}(11 \%)$ in statin use. This pilot study also examined changes in cholesterol at 6 weeks after baseline and established that the reduction in total cholesterol was greater at 6 weeks than 12 weeks, which may indicate issues with the longterm effectiveness and/or adherence to statins. ${ }^{52}$

\section{Strengths and weaknesses}

The cholesterol reduction observed in our study lies at approximately the mid-point between the two published studies outlined above, which examined rosuvastatin and pravastatin (10 and $40 \mathrm{mg}$ daily dosage, respectively). This result is consistent with estimates of the cholesterollowering activity of statins in the general population, which suggest that $20-40 \mathrm{mg}$ simvastatin (the most commonly prescribed statin in our study) has an intermediate level of activity relative to $10 \mathrm{mg}$ rosuvastatin and $40 \mathrm{mg}$ pravastatin. ${ }^{53}$ Importantly, our results provide the first evidence that cholesterol concentration is reduced by over $1 \mathrm{mmol} / \mathrm{L}$ for up to 2 years, which suggests that medication adherence is sustained. This finding is important because it suggests-in agreement with other studies $^{54}{ }^{55}$-that statin use can result in substantial reductions in cholesterol among populations with high antipsychotic usage. Furthermore, other studies in the 
general population have shown that the impact of statin prescribing on CVD events increases with extended use: reductions of $0.6 \mathrm{mmol} / \mathrm{L}$ of total cholesterol are associated with a $7 \%$ decrease in ischaemic heart disease during the first 2 years of therapy and $>20 \%$ decreases thereafter. ${ }^{56}$ The decreased total cholesterol concentrations attributed to statin therapy in people with SMI may therefore translate into long-term clinically meaningful reductions in CVD.

A limitation of our study findings for total cholesterol is that these apply only to individuals who had complete data at baseline and who had one or more measurements of total cholesterol recorded in the 2 years after the index date. Although the ascertainment of cholesterol outcome data was good for statin users (73-82\%), it is possible that individuals who are more compliant may be more likely to have a blood test. Therefore, our results may overestimate the potential effect of initiation of statin treatment. In addition, outcome data were less available for statin non-users $(55-57 \%)$. This is potentially important because, while many trials of statins suggest that cholesterol concentration is unlikely to change substantially in the control arm, long-term temporal trends in dyslipidaemia have been observed, even among those without treatment for lipid modification. ${ }^{57}$

The use of observational data for studies that compare treated and untreated arms is likely to result in comparison groups that differ, especially in terms of their risk for the condition being medicated. Although this study employed strategies to increase the comparability of statin user and non-user groups, the profiles of statin users and non-users differed at baseline, with statin users having greater CVD risk and a raised crude MI and stroke event rate. These baseline differences were anticipated and-as with other studies ${ }^{38} 40{ }^{50}$-addressed through use of staggered cohort design and adjustment for known confounders of the association between prescribing and CVD outcomes. The impact of these statistical adjustments was large and reversed the direction of association between statin prescribing and CVD events, thus highlighting the importance of confounding and the extent to which it may be controlled. However, this approach can only reduce bias arising from differences in measured confounders. Residual confounding arising both from time-varying changes in measured covariates (such as blood pressure within the baseline period) and unmeasured factors such as the severity of mental illness may still impact on the study results. In addition, although the outcomes investigated by this study have high positive predictive values, there is likely to be a small proportion $\left(\mathrm{eg}, \sim 5 \%{ }^{41}\right)$ of events that are imperfectly recorded and may therefore reduce the accuracy of our results.

The results of statin effectiveness on CVD outcomes described by this study have focused on data for the full population with imputed values of covariate data (on blood pressure, total cholesterol, smoking status, weight and height) where these measurements were unobserved at baseline. Individuals who were not prescribed a statin but who had complete baseline data made up only a small proportion $(12 \%)$ of total statin non-users included in the full analysis. It was therefore important to extend the analysis beyond individuals for whom complete data were available. We made every attempt to develop a robust approach towards imputing missing covariate data (further outlined in supplementary files) but acknowledge that our estimates are not a substitute for fully observed data. In particular, it was not possible to develop an imputation model that included high-density lipoprotein cholesterol (HDL-C) because $>90 \%$ of data were missing for HDL-C in early time periods in the study. Lipid profile (including HDL-C fraction and trigylcerides) is both predictive of CVD events and associated with SMI, through mechanisms such as poorer diet, which is associated with a lower HDL-C fraction. ${ }^{58}$ Incomplete characterisation of lipid profile within this study may result in some residual confounding, thereby potentially biasing our estimate of the effectiveness of statin prescribing, probably towards the null. The high level of similarity between results for the primary outcome using imputed data relative to complete cases (outlined in online supplementary file S4) adds greater certainty to our findings.

\section{Meaning}

The results from this study provide evidence that the potential impact of statin prescribing on intermediate outcomes in people with SMI has a magnitude that is similar to the general population. We identified statistically significant reductions in total cholesterol (of $1.2 \mathrm{mmol} / \mathrm{L}$ up to 2 years, $\mathrm{p}<0.001$ ), suggesting that medication adherence in people with SMI is sufficient to support effective lipid modification. Both CVD screening and statin prescribing should be encouraged as a potential means of decreasing the mortality gap in SMI. However, the impact of these interventions should be further evaluated relative to other interventions-such as smoking cessation-for primary prevention of CVD.

Acknowledgements The authors would like to thank John Wood (Primary Care and Population Health, UCL) for his help with sample size estimates for this study.

Contributors RB, IP, KW and DO developed the study design and protocol. RB developed the statistical code, extracted and analysed the data under the supervision of IP, KW and DO (but overseen by all co-authors) and with statistical support from MF. DO, KW and IN advised on context and clinical interpretation of the findings. RB drafted the manuscript for further development and review by all co-authors. IP is the guarantor for the study.

Funding This paper summarises independent research funded by the National Institute for Health Research (NIHR) under the Programme Grants for Applied Research (PGfAR) scheme (grant reference number RP-PG-0609-10156).

Disclaimer The views expressed are those of the author(s) and not necessarily those of the NHS, the NIHR or the Department of Health.

\section{Competing interests None declared.}

Ethics approval The THIN scheme for obtaining and providing anonymous patient data to researchers was approved by the National Health Service South-East Multicentre Research Ethics Committee (MREC) in 2002. Approval 
for this study was obtained from the Scientific Review Committee on 23 December 2014

Provenance and peer review Not commissioned; externally peer reviewed.

Data sharing statement Statistical code and medical code lists are available from the corresponding author.

Open Access This is an Open Access article distributed in accordance with the terms of the Creative Commons Attribution (CC BY 4.0) license, which permits others to distribute, remix, adapt and build upon this work, for commercial use, provided the original work is properly cited. See: http:// creativecommons.org/licenses/by/4.0/

\section{REFERENCES}

1. Osborn DP, Levy G, Nazareth II, et al. Relative risk of cardiovascular and cancer mortality in people with severe mental illness from the United Kingdom's General Practice Research Database. Arch Gen Psychiatry 2007;64:242-9.

2. Laursen TM, Munk-Olsen T, Gasse C. Chronic somatic comorbidity and excess mortality due to natural causes in persons with schizophrenia or bipolar affective disorder. PLOS ONE 2011;6: e24597.

3. Ringen PA, Engh JA, Birkenaes AB, et al. Increased mortality in schizophrenia due to cardiovascular disease-a non-systematic review of epidemiology, possible causes, and interventions. Front Psychiatry 2014;5:137.

4. National Institute for Health and Clinical Excellence. Lipid modification: cardiovascular risk assessment and the modification of blood lipids for the primary and secondary prevention of cardiovascular disease. London, UK,National Clinical Practice Guideline Number 67, 2014.

5. National Institute for Health and Clinical Excellence. Psychosis and schizophrenia in adults: treatment and management. London, UK, National Clinical Practice Guideline, Number CG178, 2014.

6. National Institute for Health and Clinical Excellence. Bipolar disorder: the assessment and management of bipolar disorder in adults, children and young people in primary and secondary care. London, UK,National Clinical Practice Guideline, Number CG185, 2014.

7. Taylor F, Huffman MD, Macedo AF, et al. Statins for the primary prevention of cardiovascular disease. Cochrane Database Syst Rev 2013;(1):CD004816.

8. Ridker PM. Rosuvastatin in the primary prevention of cardiovascular disease among patients with low levels of low-density lipoprotein cholesterol and elevated high-sensitivity C-reactive protein: rationale and design of the JUPITER trial. Circulation 2003;108:2292-7.

9. Heart Protection Study Collaborative Group. MRC/BHF Heart Protection Study of cholesterol lowering with simvastatin in 20,536 high-risk individuals: a randomised placebo-controlled trial. Lancet 2002;360:7-22

10. Shepherd J, Cobbe SM, Ford I, et al. Prevention of coronary heart disease with pravastatin in men with hypercholesterolemia. West of Scotland Coronary Prevention Study Group. N Engl J Med 1995;333:1301-7.

11. Beishuizen ED, Jukema JW, Tamsma JT, et al. No effect of statin therapy on silent myocardial ischemia in patients with type 2 diabetes without manifest cardiovascular disease. Diabetes Care 2005;28:1675-9.

12. Nakamura $\mathrm{H}$, Arakawa $\mathrm{K}$, Itakura $\mathrm{H}$, et al. Primary prevention of cardiovascular disease with pravastatin in Japan (MEGA Study): a prospective randomised controlled trial. Lancet 2006;368:1155-63.

13. Baldassarre D, Veglia C, Gobbi G, et al. Intima-media thickness after pravastatin stabilizes also in patients with moderate to no reduction in LDL-cholesterol levels: the carotid atherosclerosis Italian ultrasound study. Atherosclerosis 2000;151:575-83.

14. Owen-Smith A, Stewart C, Green C, et al. Adherence to common cardiovascular medications in patients with schizophrenia vs. patients without psychiatric illness. Gen Hosp Psychiatry 2016;38:9-14

15. Kreyenbuhl J, Dixon LB, McCarthy JF, et al. Does adherence to medications for type 2 diabetes differ between individuals with vs without schizophrenia? Schizophr Bull 2010;36:428-35.

16. Osterberg L, Blaschke T. Adherence to medication. $N$ Engl J Med 2005;353:487-97

17. Maningat $\mathrm{P}$, Gordon BR, Breslow JL. How do we improve patient compliance and adherence to long-term statin therapy? Curr Atheroscler Rep 2013;15:291.
18. Ruokoniemi P, Sund R, Arffman M, et al. Are statin trials in diabetes representative of real-world diabetes care: a population-based study on statin initiators in Finland. BMJ Open 2014;4:e005402.

19. Macklin J, Morrison G. Survey of general practitioners' attitudes to prescribing statins in different patient groups: a web-based survey. Scott Med J 2011;56:33-5.

20. Mitchell AJ, Lord O, Malone D. Differences in the prescribing of medication for physical disorders in individuals with $\mathrm{v}$. without mental illness: meta-analysis. Br J Psychiatry 2012;201:435-43.

21. Horton JD, Goldstein JL, Brown MS. SREBPs: activators of the complete program of cholesterol and fatty acid synthesis in the liver. J Clin Invest 2002;109:1125-31.

22. Ferno J, Raeder MB, Vik-Mo AO, et al. Antipsychotic drugs activate SREBP-regulated expression of lipid biosynthetic genes in cultured human glioma cells: a novel mechanism of action?

Pharmacogenomics J 2005;5:298-304.

23. Raeder MB, Ferno J, Vik-Mo AO, et al. SREBP activation by antipsychotic- and antidepressant-drugs in cultured human live cells: relevance for metabolic side-effects? Mol Cell Biochem 2006;289:167-73.

24. Nasrallah HA. Atypical antipsychotic-induced metabolic side effects: insights from receptor-binding profiles. Mol Psychiatry 2008;13:27-35.

25. IMS Health. Statistics [online]. http://www.epic-uk.org/our-data/ statistics.shtml (accessed Nov 2016).

26. Osborn DP, Hardoon S, Omar RZ, et al. Cardiovascular risk prediction models for people with severe mental illness: results from the prediction and management of cardiovascular risk in people with severe mental illnesses (PRIMROSE) research program. JAMA Psychiatry 2015;72:143-51.

27. Osborn DP, Baio G, Walters $\mathrm{K}$, et al. Inequalities in the provision of cardiovascular screening to people with severe mental illnesses in primary care: cohort study in the United Kingdom THIN Primary Care Database 2000-2007. Schizophr Res 2011;129:104-10.

28. Reilly S, Planner C, Hann M, et al. The role of primary care in service provision for people with severe mental illness in the United Kingdom. PLOS ONE 2012;7:e36468.

29. Nazareth I, King M, Haines A, et al. Accuracy of diagnosis of psychosis on general practice computer system. BMJ 1993;307:32-4.

30. Hardoon S, Hayes J, Blackburn R, et al. Recording of severe mental illness in United Kingdom Primary Care, 2000-2010. PLoS ONE 2013;8:e82365.

31. Chisholm J. The Read clinical classification. BMJ 1990;300:1092.

32. Stuart-Buttle CD, Read JD, Sanderson HF, et al. A language of health in action: Read codes, classifications and groupings. Proc AMIA Annu Fall Symp 1996:75-9.

33. Townsend $\mathrm{P}$, Phillimore $\mathrm{P}$, Beattie $\mathrm{A}$. Inequalities in health in the northern region. Newcastle upon Tyne: Northern Regional Health Authority and University of Bristol, 1986

34. Horsfall L, Walters K, Petersen I. Identifying periods of acceptable computer usage in primary care research databases. Pharmacoepidemiol Drug Saf 2013;22:64-9.

35. Maguire A, Blak BT, Thompson M. The importance of defining periods of complete mortality reporting for research using automated data from primary care. Pharmacoepidemiol Drug Saf 2009;18:76-83.

36. Lewis JD, Bilker WB, Weinstein RB, et al. The relationship between time since registration and measured incidence rates in the General Practice Research Database. Pharmacoepidemiol Drug Saf 2008;18:76-83.

37. Gagne J, Polinski J, Avorn J, et al. Standards for causal inference methods in analyses of data from observational and experimental studies in patient-centred outcomes research. Final Technical Report, 2012.

38. Seeger JD, Kurth T, Walker AM. Use of propensity score technique to account for exposure-related covariates: an example and lesson. Med Care 2007;45:S143-8.

39. Danaei G, Rodriguez LA, Cantero OF, et al. Observational data for comparative effectiveness research: an emulation of randomised trials of statins and primary prevention of coronary heart disease. Stat Methods Med Res 2013;22:70-96.

40. Hernan MA, Alonso A, Logan R, et al. Observational studies analyzed like randomized experiments: an application to postmenopausal hormone therapy and coronary heart disease. Epidemiology 2008;19:766-79.

41. Garcia Rodriguez LA, Tacconelli S, Patrignani P. Role of dose potency in the prediction of risk of myocardial infarction associated with nonsteroidal anti-inflammatory drugs in the general population J Am Coll Cardiol 2008;52:1628-36. 
42. Garcia Rodriguez LA, Varas-Lorenzo C, Maguire A, et al. Nonsteroidal antiinflammatory drugs and the risk of myocardial infarction in the general population. Circulation 2004;109:3000-6.

43. Ruigomez A, Martin-Merino E, Rodriguez LA. Validation of ischemic cerebrovascular diagnoses in the health improvement network (THIN). Pharmacoepidemiol Drug Saf 2010;19:579-85.

44. Gaist D, Wallander MA, Gonzalez-Perez A, et al. Incidence of hemorrhagic stroke in the general population: validation of data from The Health Improvement Network. Pharmacoepidemiol Drug Saf 2013;22:176-82.

45. Hall GC. Validation of death and suicide recording on the THIN UK primary care database. Pharmacoepidemiol Drug Saf 2009:18:120-31.

46. White IR, Royston P, Wood AM. Multiple imputation using chained equations: issues and guidance for practice. Stat Med 2011;30:377-99.

47. White IR, Royston P. Imputing missing covariate values for the Cox model. Stat Med 2009;28:1982-98.

48. Huber P. The behavior of maximum likelihood estimates under nonstandard conditions. 1st edn. Berkeley, California: University of California Press, 1967:221-33.

49. Smeeth L, Douglas I, Hall AJ, et al. Effect of statins on a wide range of health outcomes: a cohort study validated by comparison with randomized trials. Br J Clin Pharmacol 2009;67:99-109.

50. Danaei G, Tavakkoli M, Hernan MA. Bias in observational studies of prevalent users: lessons for comparative effectiveness research from a meta-analysis of statins. Am J Epidemiol 2012;175:250-62.
51. De Hert M, Kalnicka D, van Winkel R, et al. "Treatment with rosuvastatin for severe dyslipidemia in patients with schizophrenia and schizoaffective disorder". J Clin Psychiatry 2006;67:1889-96.

52. Vincenzi B, Stock S, Borba CP, et al. A randomized placebo-controlled pilot study of pravastatin as an adjunctive therapy in schizophrenia patients: effect on inflammation, psychopathology, cognition and lipid metabolism. Schizophr Res 2014;159:395-403.

53. Weng TC, Yang YH, Lin SJ, et al. A systematic review and meta-analysis on the therapeutic equivalence of statins. J Clin Pharm Ther 2010;35:139-51.

54. Ojala K, Repo-Tiihonen E, Tiihonen J, et al. Statins are effective in treating dyslipidemia among psychiatric patients using second-generation antipsychotic agents. J Psychopharmacol 2008;22:33-8.

55. Heald AH, Martin JL, Payton T, et al. Changes in metabolic parameters in patients with severe mental illness over a 10-year period: a retrospective cohort study. Aust N Z J Psychiatry 2017:51:75-82.

56. Law MR, Wald NJ, Rudnicka AR. Quantifying effect of statins on low density lipoprotein cholesterol, ischaemic heart disease, and stroke: systematic review and meta-analysis. BMJ 2003;326:1423

57. Muntner P, Levitan EB, Brown TM, et al. Trends in the prevalence, awareness, treatment and control of high low density lipoprotein-cholesterol among United States adults from 1999-2000 through 2009-2010. Am J Cardiol 2013;112:664-70.

58. Meyer JM, Stahl SM. The metabolic syndrome and schizophrenia. Acta Psychiatr Scand 2009;119:4-14. 